\title{
Kranioplastiken: Indikationen und Materialien
}

Thomas Kapapa, Christian Rainer Wirtz, Peter Richter

\section{Historisches}

\begin{abstract}
Merke
Erste Hinweise für die intendierte chirurgische Schädeleröffnung bzw. Behandlung von Schädelverletzungen wurden an Schädelfunden von etwa 2000 bis 400 v. Chr. in Peru gefunden [1-4].
\end{abstract}

Obgleich die Operateure noch nicht Neurochirurgen genannt wurden und nicht immer klar ist, ob ein medizinisch-kurativer Ansatz oder ein religiöses Ritual zugrunde lag, scheinen einige Patienten den Eingriff längere Zeit überlebt zu haben [5,6]. Überwiegend kam es hierbei zu knochenentfernenden Prozeduren (osteoklastische Trepanation), wahrscheinlich nach Frakturen oder intrakraniellen Blutungen. Die Kranioplastik, als Verschluss eines Schädeldefekts, wurde in vielen verschiedenen Völkern, auf Kontinenten und in Ländern, wie Polynesien, Afrika, Asien und Britannien, angewendet [7].

Oft spiegelten die Werkstoffe, die zur Kranioplastik verwendet wurden, die gesellschaftliche und sozioökonomische Stellung des Menschen wider. Fallopius war einer der Ersten, der im 16. Jahrhundert einen Unterschied in der Anwendung einer Kranioplastik bei intakter und verletzter Hirnhaut sah [7]. Einer der ältesten europäischen Hinweise für einen Verschluss eines Schädeldefekts ist ins 17. Jahrhundert datiert. Hier wurden in der Größe des Schädeldefekts korrespondierende Gold- und Silberplatten neben den Toten in Gräbern gefunden. Die erste dokumentierte xenograft-basierte Kranioplastik erfolgte etwa 1670 durch J. van Meekren, der erfolgreich Knochen eines Hundes verwendete [8,9]. Trotz des nachweislichen medizinischen Erfolgs verlangte die katholische Kirche die Explantation des Implantats.

\section{Merke}

Mit Fortschritten in der Medizin, besonders in Kriegszeiten mit einem höheren Anteil von Kriegsverletzungen, stieg die Anzahl von Kranioplastiken im 19. und 20. Jahrhundert $[10,11]$.

Carbonpolymere, Alloplastiken auf Kalziumbasis, Metallimplantate oder Implantate aus Knochen wurden hier zunehmend verwendet [12].
Heutzutage sucht man auf dem Gebiet der Neurochirurgie, Hals-Nasen-Ohren-Heilkunde und plastischen Chirurgie weiterhin nach einem Werkstoff bzw. Implantat, das neben einem hohem Maß von Verträglichkeit, Anpassungsfähigkeit an den Träger z. B. in Bezug auf Härte, Gewicht und Temperaturverhalten, eine hohe Integration in und an den umgebenden Knochen zeigt. Hierbei werden neben den chirurgischen Ansprüchen der Bearbeitbarkeit ebenfalls kosmetische Ansprüche von den Patienten gestellt.

\section{Osteoklastische Trepanation, Kraniektomie}

Weiterhin hat in unserer Gesellschaft die körperliche Integrität, die Unversehrtheit, nicht nur die Rolle des evolutionären Reproduktionsvorteils, sondern auch das Vermeiden von sozialer Stigmatisierung und somit Ausgrenzung. Osteoklastische Methoden wurden in osteoplastische Techniken überführt, also in solche, die die Knochenintegrität sofort intraoperativ oder in einer 2. Sitzung wieder herstellen. Die meisten Kranioplastiken werden derzeit im sekundären Kontext nach Trauma (Sturz, Verkehrsunfall oder Gewalttaten) durchgeführt [13]. Mit steigender Anzahl von Überlebenden, auch durch Einführung osteoklastischer Techniken wie der dekompressiven Hemikraniektomie, steigt die Anzahl von Kranioplastiken [14].

Besondere Bedeutung haben die Eingriffe der Wiederherstellung der knöchernen Integrität am Schädel nach Notfalleingriffen wie dem schweren Schädel-Hirn-Trauma oder anderen, die mit einer Erhöhung des intrakraniellen Druckes einhergehen ( $\vee$ Abb. 1).

\footnotetext{
Merke

Die dekompressive Hemikraniektomie dient der raschen Senkung des erhöhten und konservativ nicht senkbaren intrakraniellen Druckes durch Entfernen großer Schädelanteile ( $\mathbf{A b b} . \mathbf{2}$ ).
}

Dies kann auch nach Trauma, Blutung oder bei Tumoren und Infarkten notwendig werden. Sie dient der raschen Reduktion des intrakraniellen Druckes und somit zur Vermeidung oder Behandlung einer Einklemmung und Sicherung der Hirndurchblutung [14]. Denn steigt der intrakranielle Druck, mindert dies bei höherem Ausmaß die 

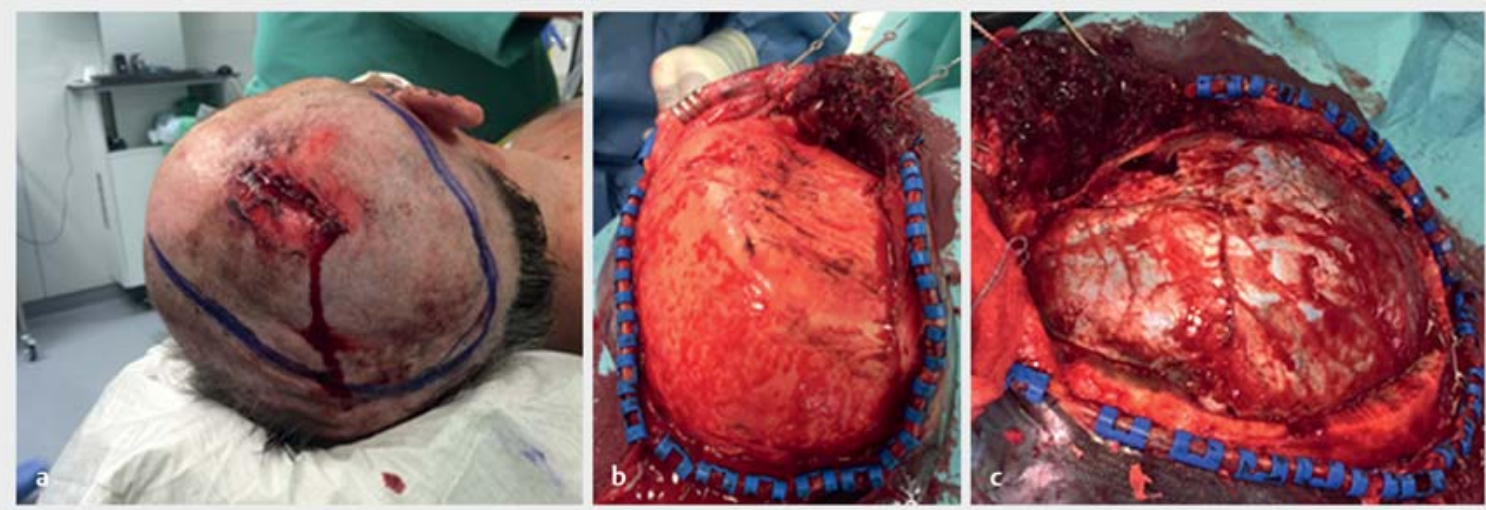

- Abb. 147 Jahre alter Patient, der auf einem Fahrrad ohne Helm fahrend von einem Pkw innerorts erfasst wurde und auf die Straße fiel. Das schwere Schädel-Hirn-Trauma zeigt sich klinisch als Koma, Anisokorie und tiefe Kopfplatzwunde links (a). Nach Entfernung der Haut und des M. temporalis links zeigt sich eine langgezogene Fraktur am Schädel (b). Nach Entfernung des großen rechtsseitigen Knochenanteils zeigt sich die Hirnhaut durch ein darunter liegendes akutes Subduralhämatom massiv gespannt (c). Aufgrund der Hirnschwellung kann der Knochen nicht intraoperativ wieder implantiert werden. Nach Hautverschluss über der adaptiv verschlossenen Dura erfolgt die Kryokonservierung des Knochens bei $-80^{\circ} \mathrm{C}$.
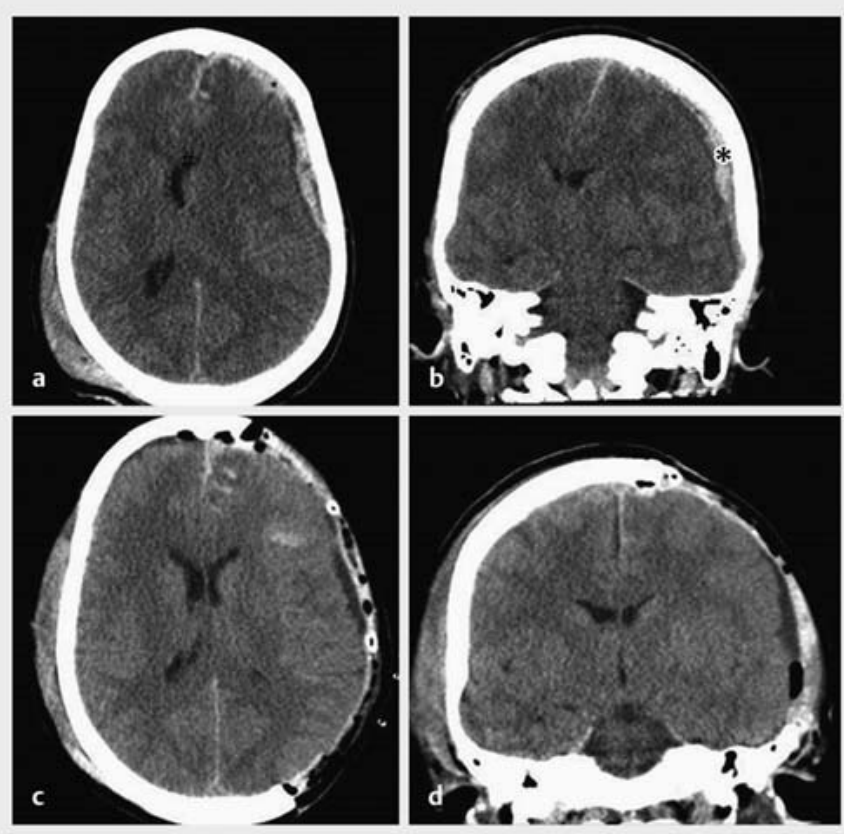

- Abb. 2 Fall einer 52-jährigen Motorradfahrerin, die mit Tempo 90 km/h auf der Autobahn verunfallte. Die Patientin wurde initial mit einem Glasgow-Coma-Score von 9 aufgefunden und intubiert. Die initiale computertomografische Abklärung zeigte eine frontale Kontusion links, ein linksseitiges akutes Subduralhämatom $\left({ }^{*}\right)$ und eine Verlagerung von Mittellinienstrukturen von $17 \mathrm{~mm}$ in axialer (a) und koronarer (b) Bildgebung. Es wurde die Indikation zur notfallmäßigen dekompressiven Hemikraniektomie gestellt. In der Kontrollbildgebung sieht man große Teile des linksseitigen Neurokraniums entfernt (c und d). Hierdurch konnte die Raumforderung durch das Hämatom beseitigt werden und dem schwellenden Hirn Platz gegeben werden, welches sich nun über die Knochenebene hernierend zeigt. Die Mittellinienstrukturen zeigen sich wieder mittelständig.
Hirnperfusion. Bislang gibt es nur für den ischämischen Hirninfarkt nach Verschluss der mittleren Hirnarterie eine Level-I-Evidenz-Empfehlung für diese chirurgische Maßnahme [15].

\section{Merke}

Zwar kann durch die dekompressive Hemikraniektomie der intrakranielle Druck gesenkt werden, in einer Vielzahl der Fälle somit auch das Überleben gesichert werden, doch bleibt das Behandlungsergebnis abhängig von der initialen Pathologie, dem Zustand des Gehirns und dem Bewusstseinszustand des Patienten [14].

Solche osteoklastischen Methoden kommen auch bei Tumoroperationen oder Operationen bei Entzündungen (autolytische Resorption) oder Infektionen (Osteomyelitis) vor. Zum Beispiel kann ein benigner Tumor wie ein Meningeom in den Schädel einwachsen und ihn in seiner Struktur destabilisieren, sodass der Tumor und der angrenzende Knochen entfernt werden muss ( $\bullet$ Abb. $\mathbf{3}$ ). Sofern sich der Lokalbefund erholt hat oder der intrakranielle Druck stabil gesenkt erscheint, kann eine Kranioplastik vollzogen werden.

Die häufig umgangssprachlich benutzte Begrifflichkeit der „Deckelung“ wird sowohl den meist lebensbedrohlichen Umständen der Kraniektomie, die ebenfalls häufig abwertend als „Entdeckelung“ betitelt wird, als auch den kosmetischen, physischen und psychischen Folgen nicht gerecht. Die Sachlage erscheint häufig viel komplexer als „Deckel runter“ oder „Deckel drauf“! Oft behalten Patienten Residuen wie eine Hemiparese oder eine Sprech- und Sehstörung zurück, die eine Wiedereingliederung in ihr altes und gewohntes Leben erschweren [16]. 


\section{Indikationen zur Kranioplastik: kosmetisch oder therapeutisch?}

Die Kranioplastik, als weitreichender Begriff, ist definiert als chirurgische Wiederherstellung der Kontinuität des (Hirn-)Schädels [17]. Hierbei spielen die Verbesserungen der Hirnfunktion und der kosmetischen Erscheinung eine wesentliche Rolle. Weiter differenziert kann eine Kranioplastik auch als Eingriff am intakten Schädel verstanden werden, womit Konturunregelmäßigkeiten, z.B. durch seitenungleiches Wachstum, behandelt werden oder auch als Augmentationseingriff.

Merke

In den meisten Fällen wird eine Kranioplastik jedoch als Ersatzverfahren für erworbene oder angeborene Schädeldefekte verstanden.

Eine Kranioplastik ist in der Lage, EEG-Veränderungen zu normalisieren, neurologische Defizite zu beseitigen und die Kognition sowie den zerebralen Blutfluss zu verbessern $[18,19]$. Grundlegende Funktion einer Kranioplastik bleibt jedoch der Schutz des darunter liegenden Hirns.

Merke

Die Indikationen zur Kranioplastik liegen im therapeutischen und kosmetischen Behandlungsfeld.

Empfohlen wird, Knochendefekte ab einer Größe von $6 \mathrm{~cm}^{2}$ zu verschließen [11]. Kleinere Knochendefekte stellen für das Hirn keine Gefahr dar, auch nicht nach Traumen, und zeigen keinen Einfluss auf den neurologischen Status. Kontraindindikationen der Kranioplastik finden sich bei Infektionen, dem Vorkommen eines Hydrozephalus und Hirnschwellung. Über den richtigen Zeitpunkt der Kranioplastik wird heftig diskutiert [6]. Dennoch ist bekannt, dass eine zu späte Kranioplastik bei Autografts und Allografts zu Infektionen sowie zu Resorption führen kann [20].

Zwar ist das Hirn nach Kraniektomie durch Hirnhaut und Haut gedeckt, doch führen der atmosphärische Druck bzw. der hydrostatische Druck bei Lagewechsel und der gestörte Fluss des Liquors dazu, dass es zu einer Depression des Hautniveaus weit unter die Knochenlücken kommen kann. Größere Defekte, besonders wenn sie die unbehaarten Anteile des Kopfes bzw. des Schädels betreffen, werden dann oft als kosmetisch unansprechend bezeichnet und empfunden. Oft kommt es bei größeren Defekten zu einem Gefühl der Schutzlosigkeit beim Patienten selbst und/oder bei Betreuern und Pflegenden. Nicht selten werden die Regionen der Kraniektomie aus Vorsicht nicht gewaschen oder gereinigt, da fälschlicherweise oft aus dem Gefühl der Unkenntnis und des Ungewohnten die Wahrscheinlichkeit der Verletzung des Gehirns durch die Knochenlücke als sehr hoch angesehen wird.
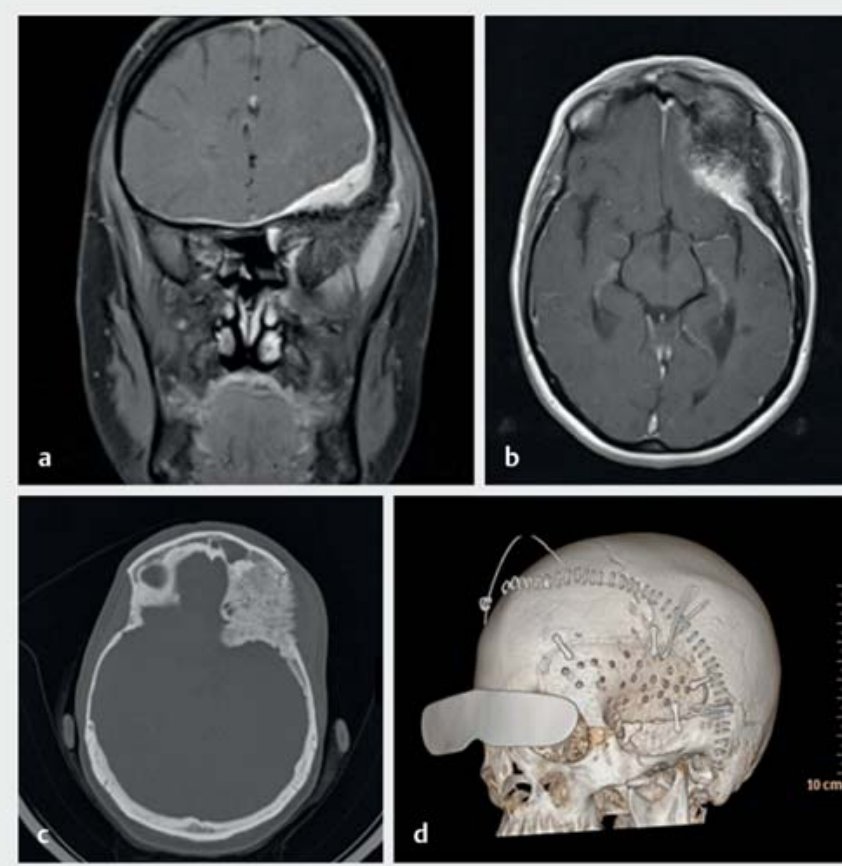

- Abb. 3 38-jährige Patientin mit einem großen Meningeom im linksseitigen frontotemporalen Schädelbereich. In der Kernspinuntersuchung ist das Meningeom ( ${ }^{*}$ ) gut mit dem Einwachsen in die Schädelbasis zu sehen ( $\mathbf{a}$ und b). Deutlicher werden die knöchernen Veränderungen in der Computertomografie (c). Postoperativ konnte ein sehr gutes kosmetisches Ergebnis erzielt werden, indem die zu resezierenden Knochenanteile präoperativ an einem Datensatz vermessen wurden, ein Implantat mittels Polymethylmethacrylat hergestellt wurde und man sich intraoperativ strikt an die Resektionsgrenzen gehalten hat. Das Implantat konnte somit passend mittels Titanplättchen in der Knochenlücke fixiert werden (d).

Neben diesen kosmetischen und pflegerischen Indikationen besteht jedoch auch eine therapeutische Indikation in Kenntnis des verbesserten klinischen Zustands nach Kranioplastik. Es gibt zahlreiche Berichte über einen Symptomrückgang oder Beschwerdebesserung nach Kranioplastik [21]. Besonders Beschwerden wie Kopfschmerzen, Müdigkeit, Reizbarkeit, Krampfanfälle oder Hemiparesen sowie psychische Beeinträchtigungen werden nach Kranioplastik in Besserung beschrieben [10].

\section{Merke}

Obgleich der Mechanismus hierfür noch gänzlich ungeklärt ist, stellt das sog. Syndrom des Trepanierten (Sinking-Skin-Flap-Syndrom, SSFS) eine Indikation zur Kranioplastik dar.

\section{Materialien zur Kranioplastik}

Historisch wurden zum Verschluss der Schädelöffnung oft andere Materialien als der eigene Knochen (autologe Kranioplastik) verwendet. Unter den nicht humanen Knochenspendern (Xenograft) sind Hunde, Kaninchen, 


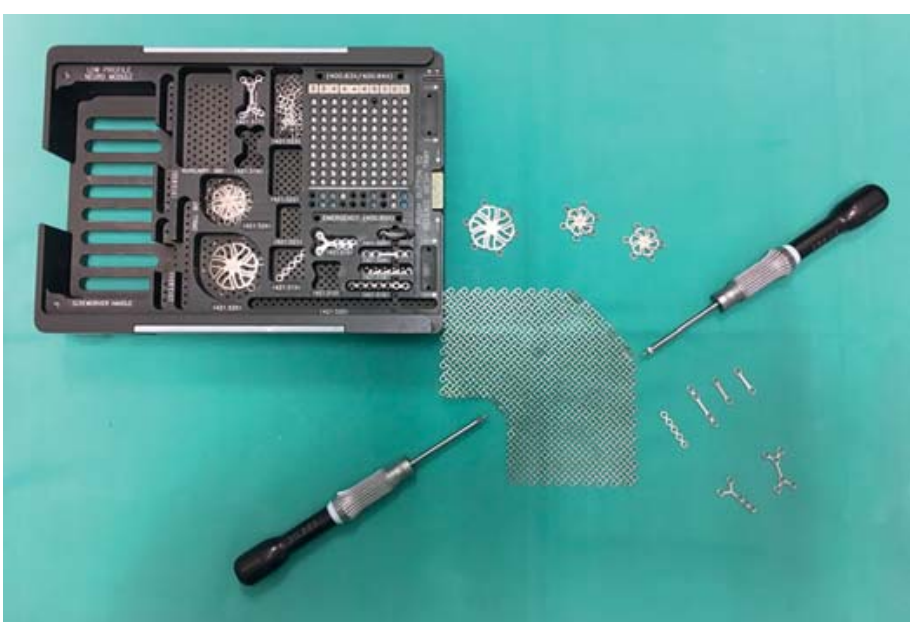

- Abb. 4 Titangitternetz im Set mit Fixierschrauben und (Steg-)Platten zum direkten Verschluss der Schädelknochenlücke oder zum Fixieren des eigenen Knochens.

Gänse, Kälber u.v.m. aufzuzählen [22]. Auch Knorpel kam zur Anwendung, da man davon überzeugt war, dass Knorpel sich dem Defekt gut anpassen würde und wenig infektanfällig sei. Es zeigte sich jedoch, dass Knorpel wenig biomechanische Stärke aufwies und eine Kalzifikation selten war [22]. Die Evolution von verschiedenen Techniken zur Kranioplastik schloss aber auch die Verwendung von (eigenen) Rippen ein. Diese erzeugten am Patienten jedoch nicht selten ein kosmetisches Ergebnis, welches als "Waschbrett" bezeichnet wurde [23]. Zur Verbesserung des kosmetischen Ergebnisses wurden dann „Spaltknochen“ oder "Split-Bone“ zur flächenhaften Deckung verwendet. Als Allograft wurde dieser Split-Bone durch verschiedene Siedetechniken in Natriumkarbonat, Alkohol und Ether hergestellt. Die hohe Rate von Infektionen und Resorptionen führte von diesem Weg wieder ab [24]. Genauso wie die Verwendung von Rippen zeigte die Verwendung von Knochen der Tibia, Os illium, Sternum, Scapula, Faszie und Fett 1889 die erschwerten Umstände in 2 Operationsgebieten: Brüche, Infektionen und Wundheilungsstörungen an Entnahmestelle und Implantationsort [18].

\section{Merke}

Ein heutiger Standard in der Neurochirurgie ist die autologe Kranioplastik.

Häufig wird der explantierte Knochen kryokonserviert oder für einen Transport des Patienten in die Bauchdecke implantiert. Am Zielort kann das Knochenstück steril wieder aus der Bauchdecke explantiert und am Schädel implantiert werden [25]. Letzteres spielt beim nicht am Ort der Primäroperation ansässigen Patienten oder dem Transport militärischer Verletzter eine bedeutende Rolle. Bei Eingriffen am Gesichtsschädel spiegeln moderne Split-Bone-Techniken ebenso einen Standard wider [26].
Die Vorteile des eigenen Knochens liegen auf der Hand: hohe Biokompatibilität, ähnliche Härte und Elastizität wie der übrige Knochen, Fähigkeit, Knochen darin einwachsen zu lassen, gute Verfügbarkeit und niedrige Kosten. Dem gegenüber stehen Nachteile wie erhöhte Anfälligkeit für Resorption, Infektanfälligkeit, eingeschränkte Bearbeitbarkeit und die Morbidität an der Entnahmestelle. Autologe Implantate heilen durch Knocheneinwachsungen bzw. schleichendes Ersetzen von Knochenzellen. Hierzu sind eine gute Blutversorgung und eine rigide Fixation notwendig. Besonders die eingeschränkte Blutversorgung bei größeren Knochendefekten und auch der lange Weg für den knöchernen Umbau spielen eine große Rolle für das Vorkommen einer Autolyse bzw. Resorption [6].

\section{Merke \\ Neben dem eigenen Knochen kommen jedoch noch andere Materialien zur Kranioplastik zur Anwendung. Aktuell werden an zu verwendende Materialien ver- schiedene Ansprüche gestellt wie Strahlendurchläs- sigkeit, Infektionsresistenz, Hitze- und Kälteunemp- findlichkeit, biomechanische Stabilität, einfache Formbarkeit zum kompletten Verschluss der Schä- dellücke, Kostengünstigkeit und Einfachheit im Gebrauch [18].}

Metalle werden seit dem frühen 19. Jahrhundert verwendet. Die Verwendung beruhte auf der Materialstärke, der Bearbeitbarkeit und auf der Sterilisierbarkeit. Im frühen 19. Jahrhundert wurde bereits Aluminium zwecks der hohen Resistenz gegenüber Infektionen verwendet. Es konnte sich jedoch nicht durchsetzen, da es häufig zur Gewebereizung, zu Krampfanfällen und zum langsamen Zerfall kam [27]. Gold hatte diese Eigenschaften nicht. Dessen Verwendung war jedoch mit anderen Erschwernissen, wie den hohen Kosten und einer relativ einfachen Verformbarkeit, verbunden. Auch Silber wurde aufgrund von Gewebereaktionen mit Silberoxid, das die Haut verfärbte, verlassen [4]. Während des Zweiten Weltkriegs kam es zur vermehrten Verwendung von Tantal. Tantal zeigte positive Eigenschaften ohne Gewebereaktion, ohne Korrosion, selten Infektionen, war inert und nicht absorbierbar [28]. Dennoch: Tantal war selten, schwer zu beschaffen und daher auch teuer und zu allem Übel speicherte es Wärme, was zu temperaturbedingten Kopfschmerzen führte [17]. Titan wird seit dem Zweiten Weltkrieg zur Kranioplastik verwendet. Eine gehäufte Anwendung fand es jedoch erst wieder in den 80er-Jahren des letzten Jahrhunderts [6]. Es kann mit Vanadium und Aluminium als Gitternetz (Mesh) alleinig oder in Verbindung mit anderen Werkstoffen wie Hydroxylapatit oder Polymethylmethacrylat (PMMA) verwendet werden ( $\bullet$ Abb. 4). Titan ist ein starker Werkstoff, der gut zu bearbeiten ist, der nicht korrodiert, wenig Gewebereaktion hervorruft und eine geringe Infektionsrate (2,6\%) aufweist [29]. 
Keramik wurde zum Ende des 20. Jahrhunderts zunehmend aufgrund der sehr hohen Stabilität, der geringen Gewebereaktion und der guten kosmetischen Ergebnisse verwendet [4]. Trotz der geringen Infektionsrate von $5,9 \%$ ergaben sich für die individuell angefertigten Keramikimplantate sehr hohe Kosten [29].

Hydroxylapatit ist ein Kalzium-Phosphat-Gemisch, das auch im gesunden Knochen gefunden wird [4]. Die synthetische Herstellung ähnelt der von Keramik. Im Gegensatz zu Metall und Acryl erlaubt Hydroxylapatit das Mitwachsen des Implantats, was besonders für Kinder eine wichtige Rolle während des Wachstums des Schädels spielt. Nachteile dieses Werkstoffs sind seine Brüchigkeit als dünnes Implantat, seine geringe Dehnungsstärke und hohe Infektionsrate [17].

Polyetheretherketon (PEEK) ist ein semikristallines Polymer, das strahlendurchlässig, inert und sterilisierbar ist [30]. Die Eigenschaften von PEEK ähneln in Stärke, Steifigkeit und Elastizität denjenigen von humanen Knochen [30]. Die heutige weite Verbreitung beruht auf der Tatsache, dass PEEK radioluzent ist und keine Artefakte in computertomografischer oder magnettomografischer Bildgebung erzeugt. PEEK ist ein sehr leichtes Material und speichert keine Wärme wie Metall oder Keramik [30]. Dennoch ist PEEK teuer und zeigt keine Integration in den umgebenden Knochen.

Acrylate (Polymethylmethacrylate, PMMA) zeigten auf den Gebieten Infektion, Korrosion, Epileptogenität und Biokompatibilität bessere Eigenschaften als Metalle. Es wird durch Anmischen eines Pulvers in eine cremige Paste überführt, die nach wenigen Minuten Bearbeitbarkeit in einer exothermen Reaktion $\left(60-80^{\circ} \mathrm{C}\right)$ aushärtet. Methyl-Methacrylat wurde 1939 entdeckt und zeigte eine fast metallene Stärke, war hitzeresistent, inert und röntgenstrahlendurchlässig [31]. Letztere Eigenschaft hatte Vorteile, da man z. B. bei Angiografien die Hirnarterien weiterhin beurteilen konnte. Nachteilig war jedoch, dass sich Brüche im Implantat kaum darstellten [32]. Hierfür wurden geringe Mengen Barium beigemischt. Auch wurden besonders für größere Kranioplastiken Titangitternetze eingewoben, um eine höhere Festigkeit zu erreichen. Weiterer Vorteil letzterer Methode war die bessere Anpassbarkeit des Methyl-Methacrylats an die anatomischen Gegebenheiten und somit ein besseres kosmetisches Ergebnis [33]. Obgleich PMMA einer der heutzutage am häufigsten benutzten Werkstoffe zur Kranioplastik ist, mit einer hohen Implantatstärke, Resistenz gegenüber Brüchen und geringer Resoprtion sowie Gewebereaktion, zeigt der Langzeitverlauf jedoch mit 12 bis $23 \%$ eine hohe Rate an Infektionen und anderen Komplikationen $[29,34]$. PMMA findet häufig Anwendung bei Erwachsenen ( $\bullet$ Abb.5). Beim wachsenden Schädel des Kindes wird es jedoch vermieden, da das Implantat kein
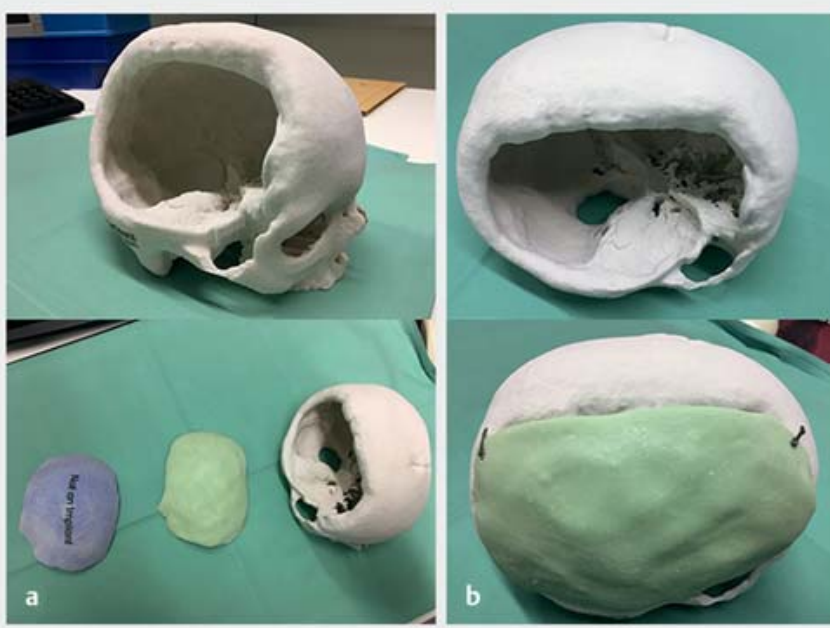

- Abb. 5 Beispielhafte Anwendung eines PMMA-Implantats. Oben der zu verschließende Knochendefekt. In (a) dargestellte Methoden zur Herstellung des Implantats mittels computerassistierten Designs. Das blaue Probeimplantat stellt den von der gesunden Schädelseite gespiegelten Knochen dar, der für die Knochenlücke berechnet wurde. Das grüne Implantat zeigt die manuell angepasste Form orientiert am explantierten Knochen oder aufgelegt auf den Situs in die Knochenlücke. In (b) dargestellte Anpassung des Implantats durch weitergehende Bearbeitung und Fixierung mittels Titanplättchen.

Ein- und somit Mitwachsen des sich verändernden Schädels aufweist.

\section{Komplikationen}

\section{Merke}

Die Komplikationen nach Kranioplastik schließen Infektionen, Wundheilungsstörungen, Knochenresorptionen, epidurale, subdurale und intrazerebrale Hämatome ein.

Hierbei spielen die zugrunde liegende Pathologie, Nebenerkrankungen und das Geschlecht eine Rolle. Das Risiko für Komplikationen nach Kranioplastik wird mit bis zu $11 \%$ beschrieben, mit dem größten Anteil für Infektionen von $6 \%$, wobei die in der Literatur berichtete Spannweite 0 bis $21 \%$ beträgt [35-37]. Ursächlicher Keim für eine Infektion ist häufig Staphylococcus aureus [37]. Ein Unterschied zwischen autologer Kranioplastik und Acrylimplantat wurde nicht festgestellt [35].

\section{Zukunftsausblick}

Mit fortschreitender computerassistierter Technologie während der Fertigungsschritte der Implantate ähneln die Werkstoffe in ihrer Form zunehmend den explantierten Knochenteilen [38]. Somit sind diese CAD-Plastiken (CAD: Computer-assisted Design) mittlerweile mit verschiede- 
nen Werkstoffen zum weiteren Standard avanciert ( $\triangleright$ Abb. 5) [6]. Die weitergehende Entwicklung beschäftigt sich zunehmend weg von Material- und Formforschung hin zu Molekular- und Zellbiologie. Weitere Verbesserungen der Applikation von Wachstumsfaktoren und Antibiotikaabgabe sind hier auch zu nennen. Die Kolonisation von multipotenten Stammzellen zur Potenzierung osteoinduktiver Prozesse auf dem Implantat scheint die Zukunft zu bedeuten [39]. Unter dem Stichwort des „Tissue Engineering“ stellen osteoinduktive Prozesse, die undifferenzierte mesenchymale Zellen in Osteoprogenitorzellen umwandeln, ein langfristiges Ziel dar [39]. Bei all diesen Ansätzen spielt die Anregung von Gefäßeinsprossung und Knochenformation in das Implantat eine große Rolle. Bei dieser Idee soll das Implantat lediglich als „Schiene“ für die Neuausbildung von Knochen dienen [18]. Diese Ansätze verfolgen einen raschen, vom Körper selbst hergestellten Verschluss der Knochenlücke mit kosmetisch ansprechendem Ergebnis und mechanischer Stärke.

\section{Interessenkonflikt}

Herr Professor Wirtz ist Berater der Firma Stryker. Bei Herrn Dr. Kapapa und Herrn Dr. Richter besteht kein Interessenkonflikt.

Über die Autoren

\section{Thomas Kapapa}

PD Dr. med. Oberarzt, Klinik für Neurochirurgie, Universitätsklinikum Ulm

\section{Christian Rainer Wirtz}

Prof. Dr. med. Direktor, Klinik für Neurochirurgie, Universitätsklinikum Ulm

\section{Peter Richter}

Dr. med. Facharzt, Klinik für Unfall, Hand-, Plastische- und Wiederherstellungschirurgie, Universitätsklinikum Ulm

\section{Korrespondenzadresse}

PD Dr. med. Oberarzt Thomas Kapapa

Klinik für Neurochirurgie

Universitätsklinikum Ulm

Albert-Einstein-Allee 23

89081 Ulm

Thomas.Kapapa@uniklinik-ulm.de

\section{Literatur}

[1] Andrushko VA, Verano JW. Prehistoric trepanation in the Cuzco region of Peru: a view into an ancient Andean practice. Am J Phys Anthropol 2008; 137: 4-13

[2] Kennedy KAR. Primitive surgery: skills before science. Am Anthropol 1987; 89: 217-218

[3] Rifkinson-Mann S. Cranial surgery in ancient Peru. Neurosurgery $1988 ; 23: 411-416$
[4] Sanan A, Haines S]. Repairing holes in the head: a history of cranioplasty. Neurosurgery 1997; 40: 588-603

[5] Bonfield CM, Kumar AR, Gerszten PC. The history of military cranioplasty. Neurosurg Focus 2014; 36: E18

[6] Goldstein JA, Paliga JT, Bartlett SP. Cranioplasty: indications and advances. Curr Opin Otolaryngol Head Neck Surg 2013; 21: $400-409$

[7] Courville CB. Cranioplasty in prehistoric times. Bull Los Angel Neuro Soc 1959; 24: 1-8

[8] Busch J. Trepanation seit dem Beginn der modernen Chirurgie. Ciba Zeitschrift 1936; 39

[9] Delashaw JB Persing JA. Repair of cranial Defects. In: Winn R, Youmans R, eds. Neurological Surgery. Philadelphia: WB Saunders; 1996: 1853-1864

[10] Gardner W]. Closure of defects of the skull with tantalum. Surg Gynecol Obstet 1945; 80: 303-312

[11] Grantham EG. Cranioplasty and the post-traumatic syndrome. J Neurosurg 1948; 5: 19-22

[12] Gladstone HB, McDermott MW, Cooke DD. Implants for cranioplasty. Otolaryngol Clin North Am 1995; 28: 381-400

[13] Dujovny M, Aviles A, Agner C et al. Cranioplasty: cosmetic or therapeutic? Surg Neurol 1997; 47: 238-241

[14] Sahuquillo J, Arikan F. Decompressive craniectomy for the treatment of refractory high intracranial pressure in traumatic brain injury. Cochrane Database Syst Rev 2006; (1): CD003983

[15] Vahedi K Hofmeijer J, Juettler E et al. Early decompressive surgery in malignant infarction of the middle cerebral artery: a pooled analysis of three randomised controlled trials. Lancet Neurol 2007; 6: 215-222

[16] Kapapa T, Brand C, Wirtz CR et al. Outcome after decompressive craniectomy in different pathologies. World Neurosurg 2016; 93: 389-397

[17] Shah AM, Jung $H$, Skirboll S. Materials used in cranioplasty: a history and analysis. Neurosurg Focus 2014; 36: E19

[18] Aydin S, Kucukyuruk B, Abuzayed B et al. Cranioplasty: Review of materials and techniques. J Neurosci Rural Pract 2011; 2: 162-167

[19] Jelcic N, Della Puppa A, Mottaran R et al. Case series evidence for improvement of executive functions after late cranioplasty. Brain Inj 2013; 27: 1723-1726

[20] Grant GA, Jolley M, Ellenbogen RG et al. Failure of autologous bone-assisted cranioplasty following decompressive craniectomy in children and adolescents. J Neurosurg 2004; 100 (2 Suppl Pediatrics): S163-S168

[21] Schorl M. Sinking skin flap syndrome (SSFS) - clinical spectrum and impact on rehabilitation. Cent Eur Neurosurg 2009; 70: 68-72

[22] Durand JL, Renier D, Marchac D. [The history of cranioplasty]. Ann Chir Plast Esthet 1997; 42: 75-83

[23] Kulali A, Kayaalp S. Single-table autogenous calvarial grafting for cranioplasty. J Craniomaxillofac Surg 1991; 19: 208-211

[24] Grant FC, Norcross NC. Repair of cranial defects by cranioplasty. Ann Surg 1939; 110: 488-512

[25] Inamasu J, Kuramae T, Nakatsukasa M. Does difference in the storage method of bone flaps after decompressive craniectomy affect the incidence of surgical site infection after cranioplasty? Comparison between subcutaneous pocket and cryopreservation. J Trauma 2010; 68: 183-187

[26] Koenig W], Donovan JM, Pensler JM. Cranial bone grafting in children. Plast Reconstr Surg 1995; 95: 1-4 
[27] Booth JA, Curtis BF. I. Report of a case of tumor of the left frontal lobe of the cerebrum; operation; recovery. Ann Surg 1893; 17: $127-139$

[28] Fulcher $\mathrm{OH}$. Tantalum as a metallic implant to repair cranial deficits. JAMA 1943; 121: 931-933

[29] Matsuno A, Tanaka H, Iwamuro $\mathrm{H}$ et al. Analyses of the factors influencing bone graft infection after delayed cranioplasty. Acta Neurochir (Wien) 2006; 148: 535-540

[30] Lethaus B, Safi Y, ter Laak-Poort M et al. Cranioplasty with customized titanium and PEEK implants in a mechanical stress model. J Neurotrauma 2012; 29: 1077-1083

[31] Woodhall B, Spurling RG. Tantalum cranioplasty for war wounds of the skull. Ann Surg 1945; 121: 649-668

[32] Henry HM, Guerrero CMoody RA. Cerebrospinal fluid fistula from fractured acrylic cranioplasty plate. Case report. J Neurosurg 1976; 45: 227-228

[33] Galicich JH, Hovind KH. Stainless steel mesh-acrylic cranioplasty. Technical note. J Neurosurg 1967; 27: 376-378

[34] Blum KS, Schneider SJ, Rosenthal AD. Methyl methacrylate cranioplasty in children: long-term results. Pediatr Neurosurg 1997; 26: 33-35
[35] Klinger DR, Madden C, Beshay J et al. Autologous and acrylic cranioplasty: a review of 10 years and 258 cases. World Neurosurg 2014; 82: e525-e530

[36] Yadla S, Campbell PG, Chitale R et al. Effect of early surgery, material, and method of flap preservation on cranioplasty infections: a systematic review. Neurosurgery 2011; 68: 11241129

[37] Bhaskar IP, Inglis T], Lee GY. Clinical, radiological, and microbiological profile of patients with autogenous cranioplasty infections. World Neurosurg 2014; 82: e531-e534

[38] Rotaru H, Stan H, Florian IS et al. Cranioplasty with custommade implants: analyzing the cases of 10 patients. J Oral Maxillofac Surg 2012; 70: e169-e176

[39] Chim H, Schantz JT. New frontiers in calvarial reconstruction: integrating computer-assisted design and tissue engineering in cranioplasty. Plast Reconstr Surg 2005; 116: 1726-1741

Bibliografie

DOI http://dx.doi.org/10.1055/s-0042-123097

OP-JOURNAL 2017; 33: 49-55 @ Georg Thieme Verlag KG Stuttgart · New York ISSN 0178-1715 